\title{
Lymphome non hodgkinien à localisation mandibulaire : à propos de deux observations
}

\author{
Non-hodgkinson lymphoma with mandibular localisation: two case histories
}

VICTORIN AHOSSI, SYLVIE BOISRAMÉ-GASTRIN, GHISLAIN PERROT, MOHSSINE TAZI, PATRICK LARRAS, DANIEL PERRIN

\section{RÉSUMÉ}

Les lymphomes sont des proliférations malignes intéressant le tissu lymphoïde, représentés principalement par le lymphomes non hodgkiniens (LNH). Le tube digestif avec la cavité buccale constitue le principal site des lymphomes non hodgkiniens extra-ganglionnaires (LNHE) [14]. De part leur fréquence et leur gravité, les LNH constituent toujours, malgré d'importants progrès thérapeutiques récents, un défi pour les onco-hématologistes.

Deux cas de LNH à grandes cellules $\mathrm{B}$, se présentant comme des tuméfactions gingivales isolées, sont rapportés. Ils illustrent le polymorphisme de l'aspect clinique des LNH dans la cavité buccale, simulant respectivement un processus infectieux d'origine odontogène et une tumeur odontogène bénigne. (Med Buccale Chir Buccale 2006; 12: 21-29).

mots clés : lymphome, cavité buccale, tumeur gingivale, lymphome malin, tissu lymphoïde associé muqueuse, pratique générale dentisterie

\section{SUMMARY}

Lymphomas are malignant proliferations involving lymphoid tissue and are principally represented by non-Hodgkinson lymphomas $(\mathrm{LNH})$. The digestive tract, along with the oral cavity, constitute the main sites of extra-ganglion nonHodgkinson-type lymphomas (LNHE) [14]. Due to their frequency and gravity, $L N H$, in spite of recent therapeutic advances, always constitute a challenge for onco-haematologists.

Two cases of $L N H$ with large $B$ cells presenting with isolated gingival tumefactions are described. They illustrate the polymorphism of the clinical aspects of $L N H$ in the oral cavity, simulating respectively an infectious process of odontogenic origin and a benign odontogenic tumour. (Med Buccale Chir Buccale 2006; 12: 21-29).

key words : lymphoma, oral cavity, gingival tumour, malignant lymphoma, lymphoid tissue, associated mucosa, the practice of general dentistry

\footnotetext{
* Service d'odontologie - C.H.U. Dijon

Demande de tirés à part :

Victorin Ahossi Service d'Odontologie CHU de Dijon 3 rue Faubourg Raines 21000 Dijon France victorin.ahossi@chu-dijon.fr
} 
Les lymphomes non hodgkiniens (LNH), proliférations malignes intéressant le tissu lymphoïde, représentent la majorité des lymphomes. La nouvelle classification des lymphomes de l'Organisation Mondiale de la Santé (OMS) de 2001 définit une quarantaine d'entités en dehors du lymphome hodgkinien. Le LNH est actuellement l'hémopathie maligne la plus fréquente avec un taux annuel standardisé à la population mondiale de 6,7/100 000 habitants par an [15]. Les lymphomes diffus à grandes cellules $\mathrm{B}$ constituent le prototype des lymphomes dits agressifs, caractérisés à la fois par une évolution spontanée rapidement fatale mais aussi par une possibilité de guérison après traitement [1]. Deux cas de LNH diffus à grandes cellules $B$, à localisation mandibulaire, ainsi que leur évolution sont présentés.

médecine

buccale

chirurgie

buccale

VOL. $12, \mathrm{~N}^{\circ} 1$ 2006

page 22

\section{OBSERVATIONS}

La première observation concerne une femme de 47 ans, sans antécédents médico-chirurgicaux majeurs, adressée par son odontologiste traitant pour une masse gingivale associée à une tuméfaction submandibulaire persistant malgré une antibiothérapie prolongée (Fig. 1). L'examen clinique initial met en évidence une lésion gingivale ferme, indolore, de $2 \mathrm{~cm}$ de diamètre, englobant les racines résiduelles de 35, 36, 37 (Fig. 2 et 3). L'état bucco-

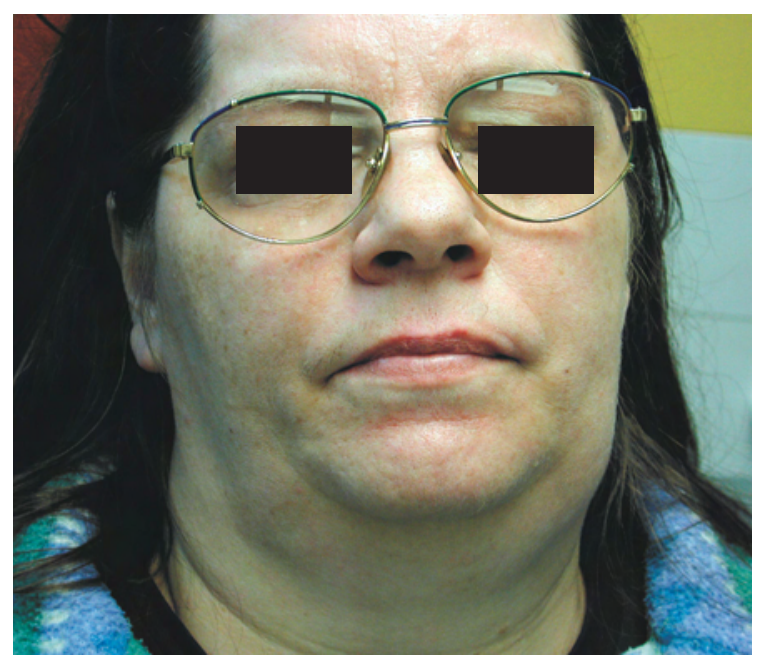

Figure 1 : Tuméfaction sous-mandibulaire gauche Left submandibular swelling dentaire est déplorable. Une adénopathie ferme, mobile, indolore, homolatérale complète le tableau clinique. La patiente signale par ailleurs une paresthésie labio-mentonnière homolatérale. Les examens complémentaires (orthopantomogramme et examen tomodensitométrique mandibulaire) ne révèlent aucune atteinte osseuse en dehors de l'élargissement du canal mandibulaire et du fora-

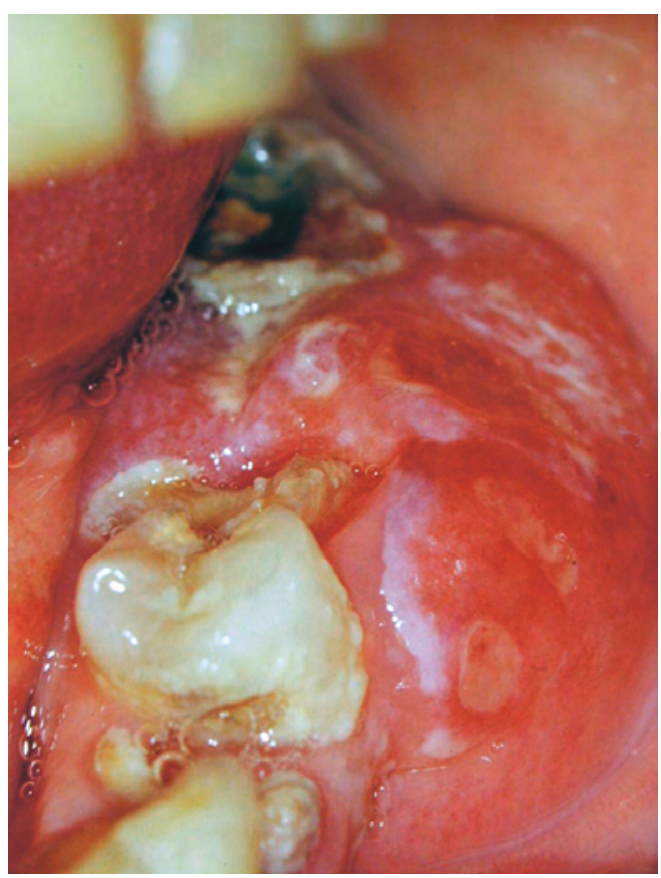

Figure 2 : Tuméfaction gingivale et restes radiculaires Gingival swelling with root debris

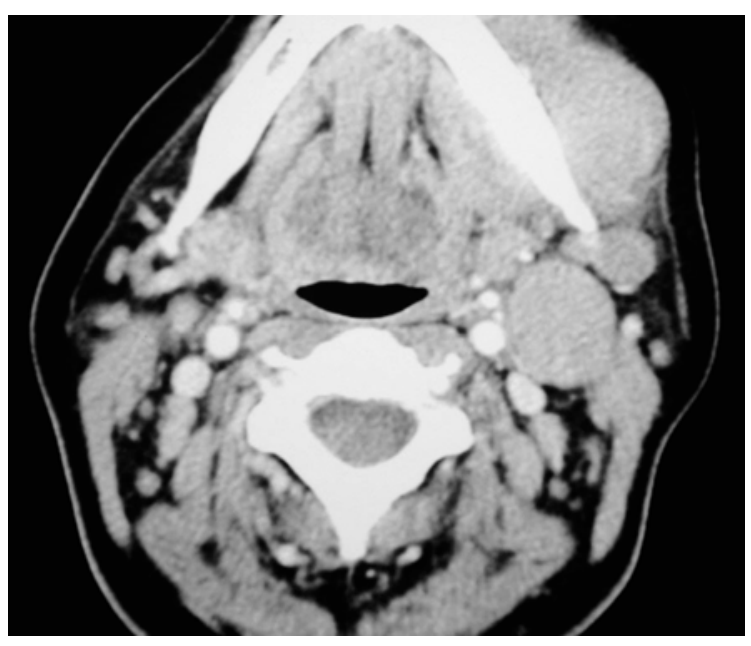

Figure 3 : Examen tomodensitométrique visualisant la masse tumorale

CT scan showing the tumour mass 
men mentonnier (Fig. 4 et 5). La biopsie gingivale met en évidence, après coloration à l'hématoxyline - éosine - safran, un infiltrat lymphoïde dense et diffus, constitué par une prolifération de grandes cellules lymphoïdes, composées de centroblastes et d'immunoblastes (Fig. 6). Le marquage anti-CD20 de toutes les cellules confirme le diagnostic de lymphome $\mathrm{B}$ diffus à grandes cellules, centroblastique polymorphe (Fig. 7). Le bilan biologique retrouve

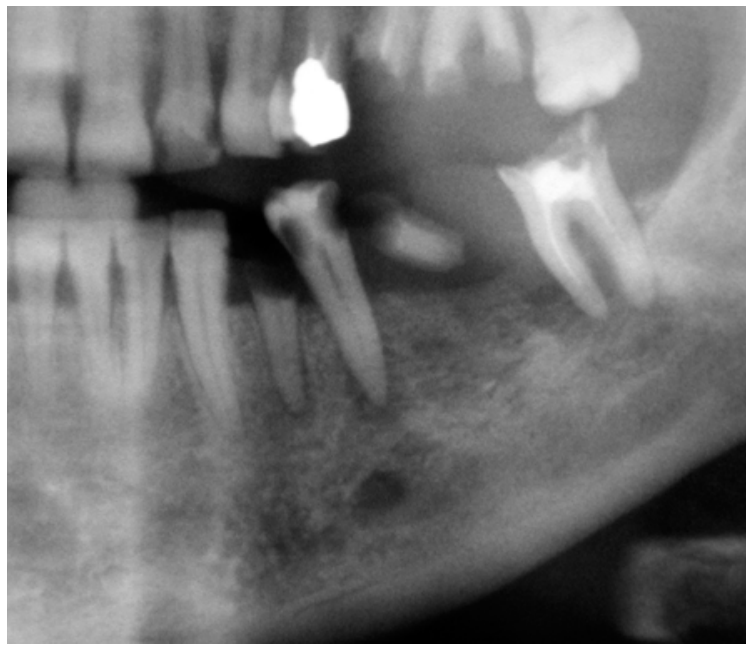

Figure 4 : Orthopantomogramme initial : élargissement du foramen mentonnier Initial panoramic X-ray: enlargement of the mental foramen

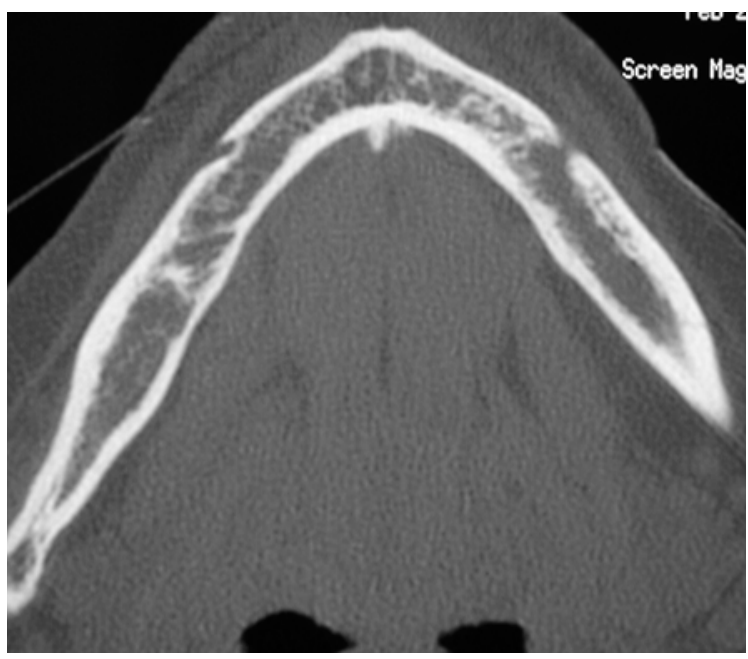

Figure 5 : Elargissement du canal mandibulaire Widening of mandibular channel

une augmentation de la lacticodéshydrogénase (LDH), véritable marqueur de la prolifération tumorale. Le bilan d'extension, par examen tomodensitométrique corps entier, révèle des adénopathies lombo-aortiques et iliaques associées à de multiples localisations intra-spléniques. II existe par ailleurs un envahissement médullaire massif de stade IV. Malgré l'état bucco-dentaire déplorable, et après concertation avec les oncologues, on opte pour une

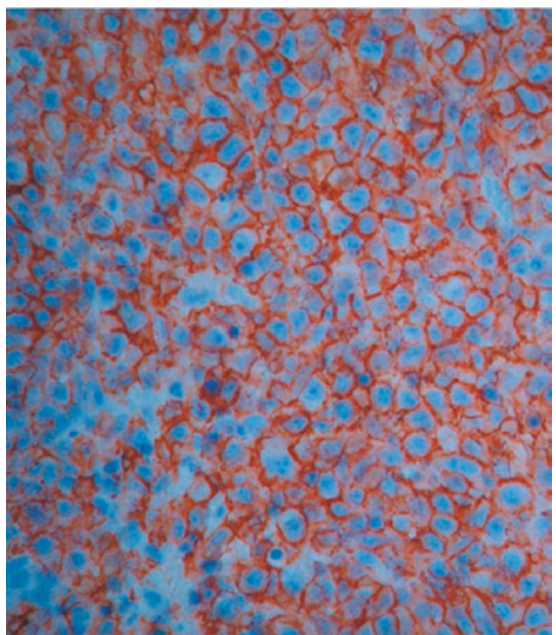

Figure 6 : Anatomopathologie (coloration HES) : prolifération de grandes cellules lymphoïdes composées de centroblastes et immunoblastes

Histology (HES dye): proliferation of large lymphoid cells (consisting) of centroblasts and immunoblasts

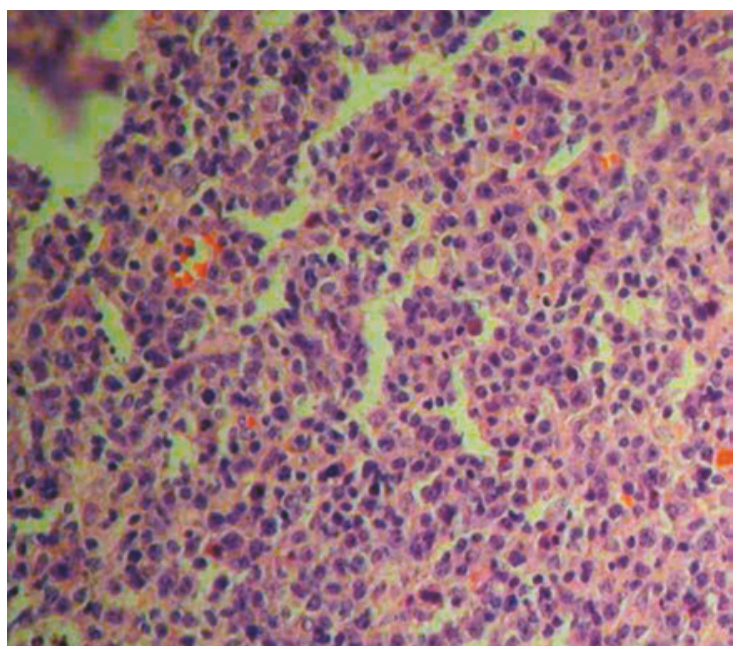

Figure 7 : Marquage par l'anticorps anti-CD20 de toutes les cellules confirmant le diagnostic de lymphome diffus à grandes cellules $\mathrm{B}$

Marking of all the cells (using antibody anti-CD20) confirming the diagnosis of diffuse lymphoma with large $B$ cells médecine

buccale

chirurgie

buccale

VOL. $12, \mathrm{~N}^{\circ} 1$ 2006

page 23 
abstention thérapeutique locale initiale afin d'éviter toute surinfection. La patiente a bénéficié dans un premier temps d'une polychimiothérapie d'induction, suivie d'une intensification avant de réaliser l'autogreffe de cellules souches périphériques. Ce traitement a donné une excellente réponse. Les avulsions ont été réalisées avant l'autogreffe et une parfaite cicatrisation a été obtenue (Fig. 8 et 9). Trois ans après la fin du traitement, la patiente est toujours en rémission. Elle ne présente aucune adénopathie, ni hépatomégalie. L'auscultation cardiopulmonaire, le bilan biologique et l'examen tomodensitométrique cervico-abdomino-pelvien sont normaux.

La deuxième observation concerne un homme de 47 ans, sans antécédents médico-chirurgicaux évocateurs, adressé par son praticien pour une tumé-

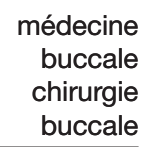

VOL. $12, \mathrm{~N}^{\circ} 1$ 2006

page 24

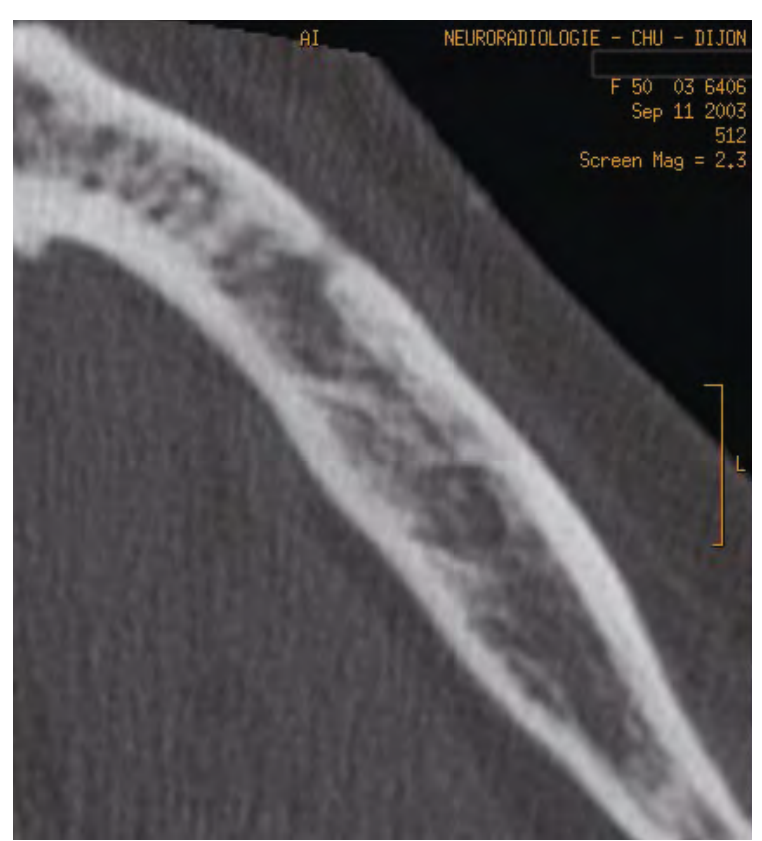

Figure 8 : Cicatrisation osseuse après polychimiothérapie. Photographie prise après deux ans de rémission Osseous healing following chemotherapy. Image taken after 2 years remission faction gingivale d'apparition brutale. L'examen clinique révèle une tuméfaction ferme, asymptomatique, sur le versant vestibulaire de la symphyse mandibulaire (Fig. 10). L'orthopantomogramme et l'examen tomodensitométrique mandibulaire ne mettent en évidence aucune image de lyse osseuse (Fig. 11). L'examen histopathologique de la biopsie exérèse de la lésion révèle un lymphome diffus à grandes cellules, de haut grade de malignité (Fig. 12, 13 et 14). Le malade est donc adressé au centre de cancérologie où un bilan d'extension est pratiqué. La biopsie médullaire révèle un envahissement de stade I. Quelques jours après la biopsie, il n'y a pas de signe de récidive. Une cure de chimiothérapie est initiée et la régression complète de la lésion persiste depuis la fin du traitement il y a seize mois (Fig. 15).

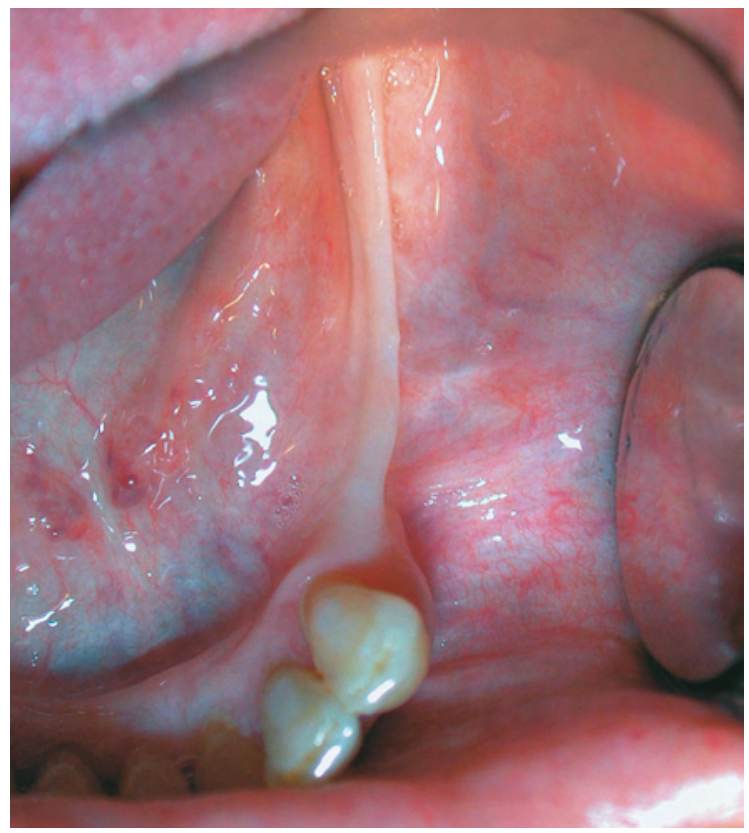

Figure 9 : Cicatrisation de la muqueuse après polychimiothérapie et avulsions

Cicatrization of the mucous membrane after polychemotherapy and extractions 


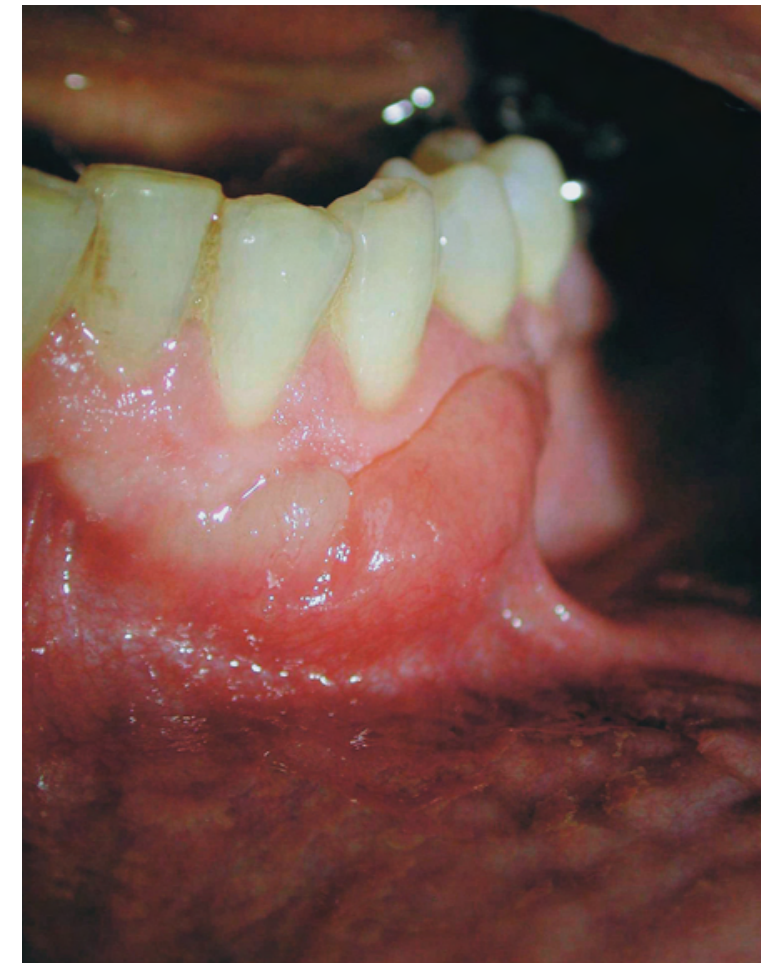

Figure 10 : Aspect clinique endobuccal Clinical oral view

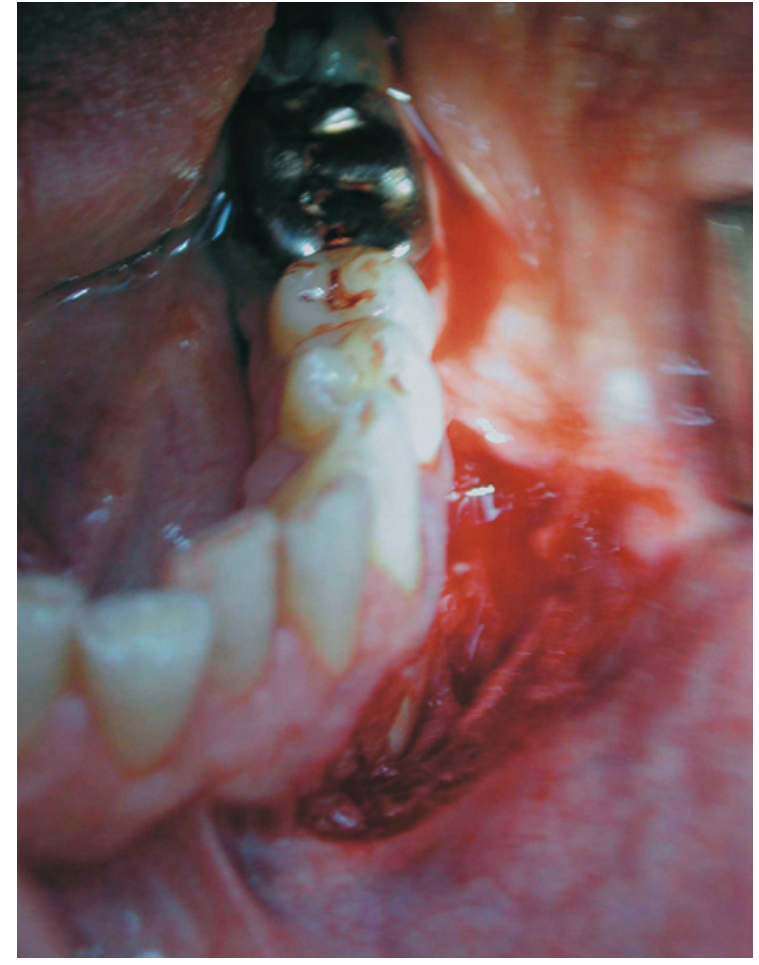

Figure 12 : Biopsie exérèse de la lésion Surgical biopsy of the lesion médecine

buccale

chirurgie

buccale

VOL. $12, \mathrm{~N}^{\circ} 1$

2006

page 25

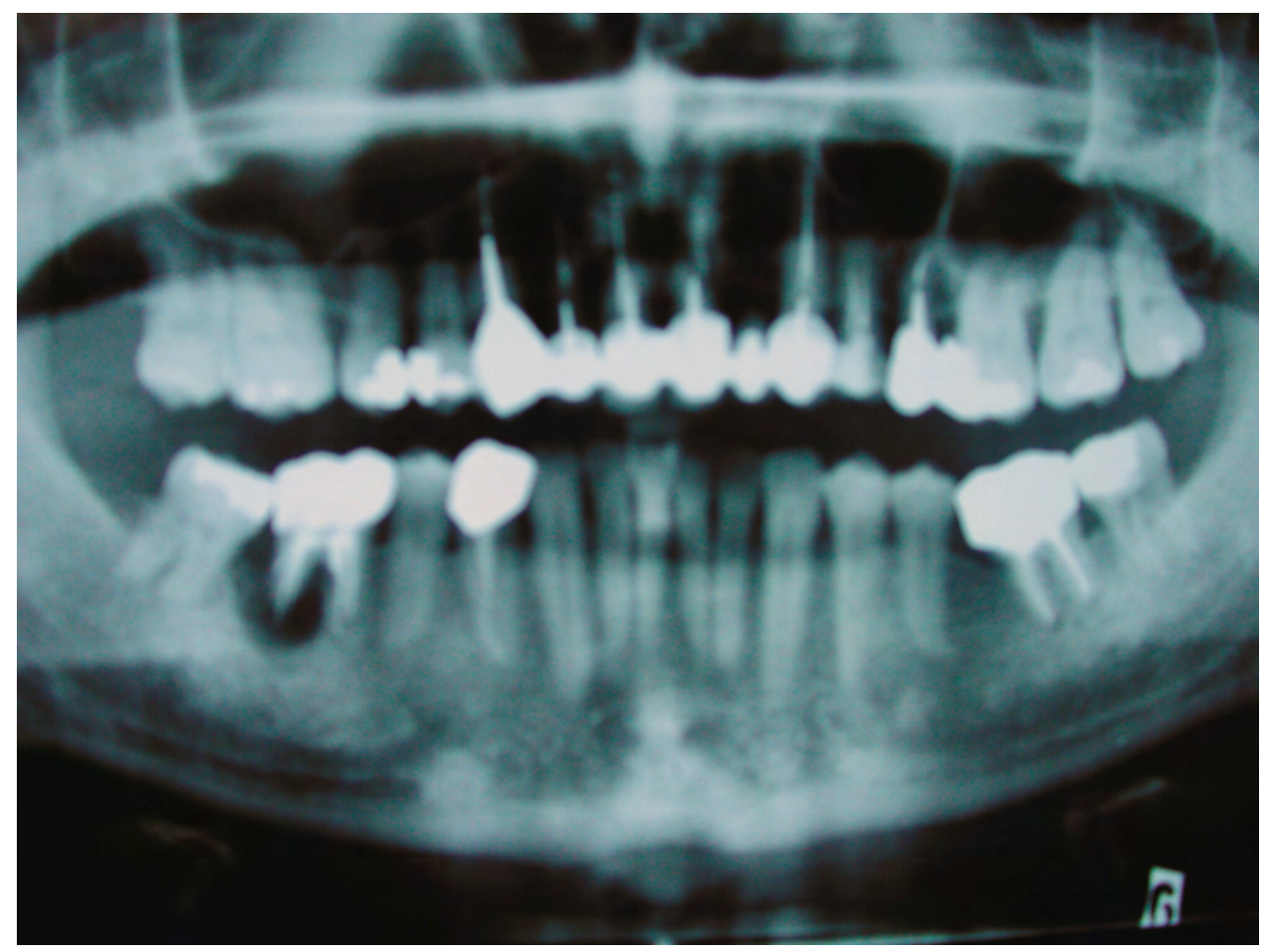

Figure 11 : Orthopantomogramme initial Initial panoramic X-ray 


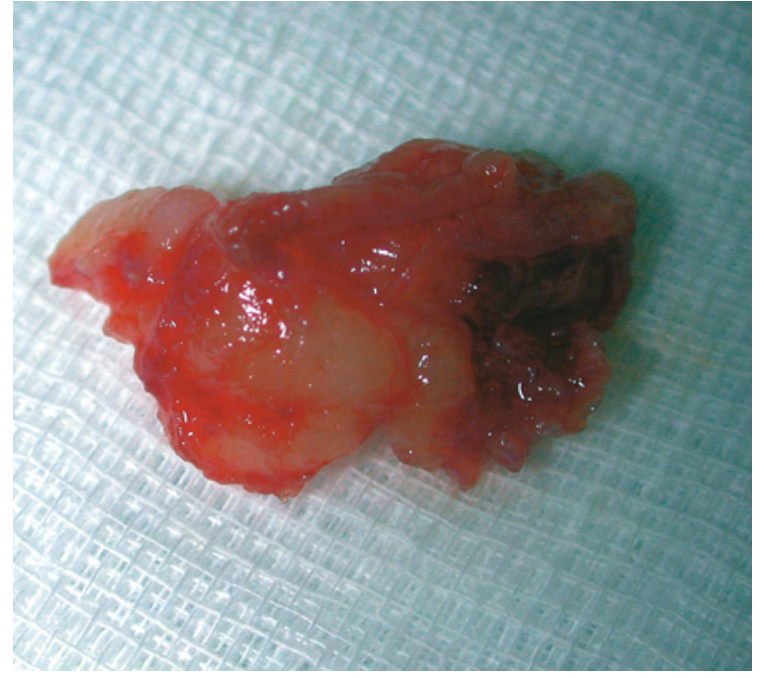

Figure 13: Pièce d'exérèse Biopsy

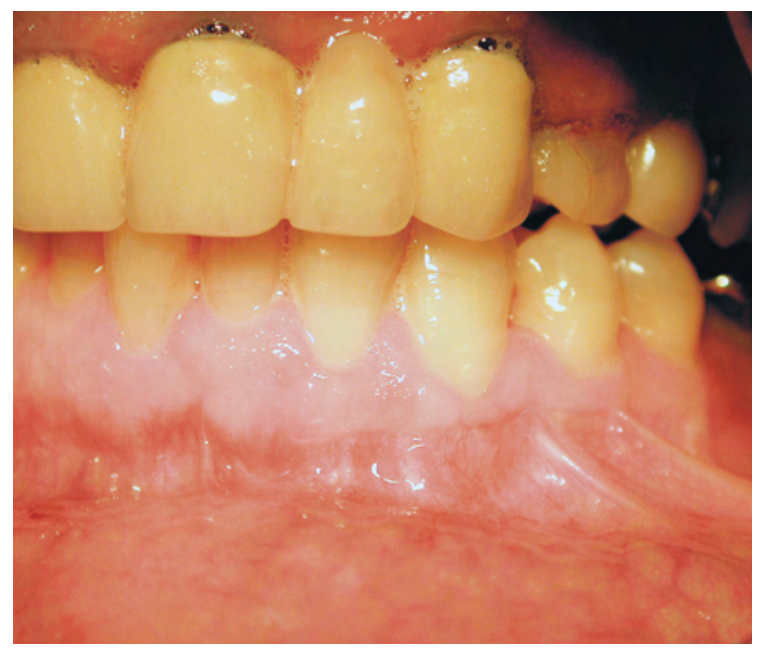

Figure 15 : Cicatrisation gingivale seize mois après la fin du traitement.

Gingival healing sixteen months after the end of treatment

\section{COMMENTAIRES}

Les LNH sont des proliférations cellulaires clonales malignes, intéressant le tissu lymphoïde. Selon le type observé, l'aspect des cellules est très variable, allant de celui de cellules souche à celui de cellules presque différenciées. L'OMS en définit une quarantaine de formes [3]. Le diagnostic peut s'avérer difficile lorsqu'il s'agit d'une atteinte extra-ganglionnaire isolée. Avec plus de 7000 nouveaux cas par an en France et une aug-

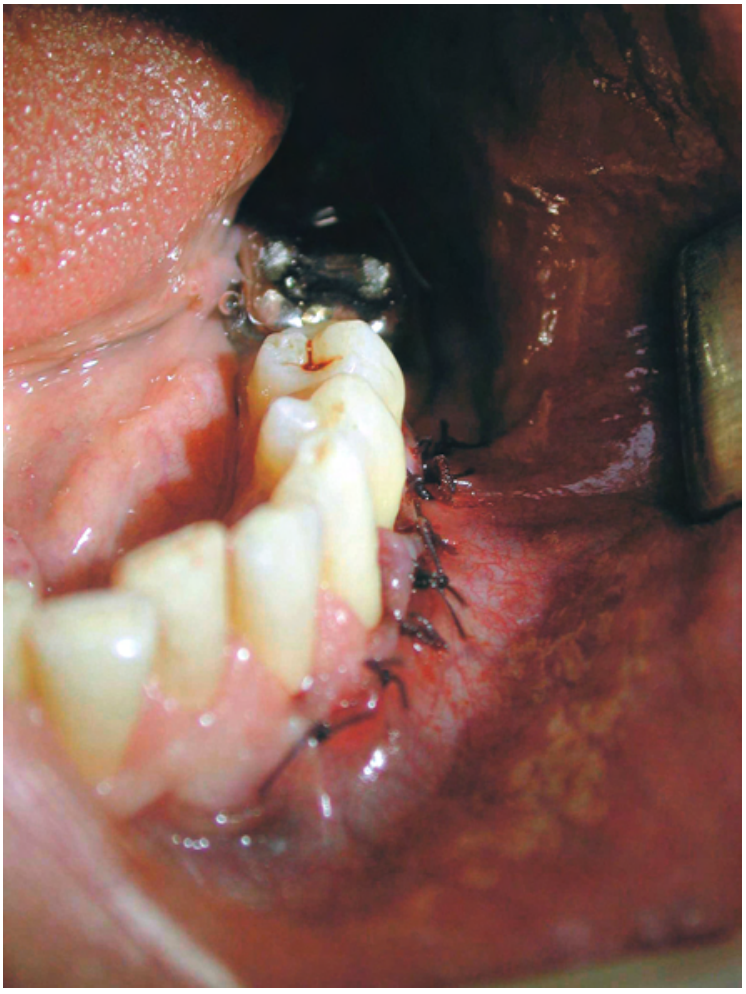

Figure 14 : Sutures résorbables Resorbable sutures

mentation de l'incidence de $4 \%$ par an, les LNH sont maintenant aussi fréquents que les mélanomes [2]. Comme pour l'ensemble des hémopathies, il existe une prédominance masculine avec un sex-ratio de 1,5 homme pour une femme. De par leur fréquence et leur gravité, les LNH constituent toujours, malgré d'importants progrès thérapeutiques récents, un défi pour les oncohématologistes. Les LNH agressifs sont caractérisés à la fois par une évolution spontanée rapidement fatale mais aussi par une possibilité de guérison après traitement. $60 \%$ des LNH correspondent à l'entité histopathologique « diffus à grandes cellules B » [1].

Cinq facteurs de pronostic sont retenus pour les LNH agressifs : I'âge (inférieur ou égal à 60 ans versus plus de 60 ans), l'index d'activité du patient selon l'échelle de l'ECOG (Eastern Cooperative Oncology Group), le stade selon Ann Arbor (I et II versus III et IV), le taux sérique de la LDH et le nombre de localisations extra-ganglionnaires (0-1 versus 2 et plus). 
Ces deux observations illustrent le polymorphisme de l'expression clinique des LNH dans la cavité buccale : dans le premier cas, il simule un processus infectieux odontogène, dans le deuxième cas une tumeur odontogène bénigne.

Les atteintes révélatrices des LNH ont dans $30 \%$ des cas un siège extra-ganglionnaire [1] et constituent les LNH extra-ganglionnaires (LNHE) qui s'observent en premier lieu dans la région cervico-faciale (30 à $40 \%$ des cas) [5], puis dans l'estomac [1]. Les LNH associés à l'anneau de Waldeyer sont bien connus et souvent classés dans le groupe de LNH associés aux tissus lymphoïdes muqueux ou lymphomes MALT (Mucosa Associated Lymphoïd Tissue) [13]. Les LNHE buccaux sont rares et touchent habituellement le palais, la mandibule, la muqueuse buccale, le plancher buccal et la gencive [9]. Les LNHE peuvent affecter à la fois l'os et les tissus mous mais siégent le plus souvent sur le palais et la mandibule [9]. II est cependant rare de trouver des LNHE à localisation gingivale [4].

Dans une étude portant sur 116 LNHE à localisation cervico-faciale, la grande majorité des cas intéresse la région amygdalienne, seulement $7,8 \%$ l'os ou la gencive ; à peine $6 \%$ se développent au palais [6]. La mandibule est plus souvent atteinte que le maxillaire. L'âge moyen lors de la découverte est de 57 ans, et il existe avec une prédominance masculine.

Devant une tumeur gingivale, plusieurs diagnostics peuvent être évoqués. Après avoir éliminé une tumeur bénigne, on doit écarter un carcinome épidermoïde ou plus rarement une tumeur maligne d'origine conjonctive. Par exemple Lynch et coll. rapportent le cas d'un patient présentant une masse gingivale exophytique due à un sarcome granulocytaire. Lors du bilan, on découvre une masse endobronchique qui se relève un lymphome à cellules $B$ [7]. Enfin, il est intéressant de savoir que le cancer primaire qui métastase le plus communément dans la gencive est le cancer du poumon, notamment chez des hommes âgés [7].

Les LNH peuvent toucher tous les organes. Les techniques d'imagerie sont donc importantes, en particulier l'examen tomodensitométrique corps entier qui permet de mettre rapidement en évi- dence les différentes localisations. L'élargissement du canal mandibulaire est rarement observé. Yamada et coll. rapportent le cas d'une patiente présentant un élargissement du canal mandibulaire sans paresthésies labiales, associé à un LNH à petites cellules ; l'élargissement du canal mandibulaire semblait être secondaire à une sclérose osseuse périphérique [11]. Robbins et coll. ont observé un élargissement du foramen mentonnier accompagné d'images radiostransparentes chez un patient atteint d'un lymphome mandibulaire [12]. L'hyperesthésie, parfois observée dans le territoire nerf mentonnier, peut être secondaire à une compression ou à une infiltration du nerf alvéolaire inférieur [11]. Dans les lésions osseuses primitives, la douleur est souvent présente, accompagnée d'une tuméfaction et de paresthésies labio-mentonnières lorsque le lymphome siège dans la branche horizontale de la mandibule. Tardivement une fracture spontanée peut survenir. Les dents deviennent parfois mobiles sans atteinte parodontale évidente. Dans certains cas, il existe des algies dentaires sans cause apparente qui sont secondaires à l'infiltration de la pulpe dentaire par les cellules lymphomateuses [16]. Des adénopathies cervicales sont fréquemment présentes. Les signes radiologiques sont pauvres : l'ostéolyse, mal délimitée, est sans rapport avec l'atteinte microscopique des lésions. Parfois une ostéocondensation est observée à la périphérie de la tumeur. Cet aspect radiologique et la présence de troubles sensitifs font plutôt évoquer une ostéomyélite [17].

L'étiopathogénie des LNH n'est pas clairement élucidée [9], et des facteurs multiples sont évoqués [15] : une origine clonale (uni ou multicentrique), une anomalie chromosomique, une déficience biochimique (enzymatique), une infection virale $(\mathrm{VIH}, \mathrm{VEB}, \mathrm{VHH} 8)$, des facteurs liés à l'environnement (radiations, facteurs toxiques) et des facteurs génétiques (raciaux, familiaux) [15]. Les rétrovirus favorisent le développent de cancers par des mécanismes directs (dysrégulation de la croissance cellulaire) et indirects (interaction avec les signaux cellulaires, induction de facteurs de croissance, perturbation de l'immunité de l'hôte). La diminution du répertoire des cellules $\mathrm{T}$, la perte du contrôle immunologique des médecine

buccale

chirurgie

buccale

VOL. $12, \mathrm{~N}^{\circ} 1$ 2006

page 27 
médecine

buccale

chirurgie

buccale

VOL. $12, \mathrm{~N}^{\circ} 1$ 2006

page 28 cellules, de même que certains virus comme le VEB, favorisent l'émergence de néoplasies. Les lymphomes MALT peuvent ainsi être associés à des désordres auto-immuns comme le syndrome de Sjögren ou la maladie d'Hashimoto [10] ; les lymphomes MALT gastriques sont très particuliers puisque leur développement semble favorisé par une infection prolongée par Helicobacter pylori [19]

La cavité buccale représente un site naturel de réplication pour le VEB. II est associé au développement des lymphomes buccaux chez les patients atteints du SIDA où il favorise la transformation maligne des lymphocytes B [9]. II est aussi associé au lymphome de Burkitt, observé principalement chez les enfants d'Afrique centrale. Le VHH 8 présente également un lymphotropisme. Même si les données sont controversées, il semble que la présence du VHC pendant de très nombreuses années dans l'organisme peut, par une stimulation prolongée des lymphocytes $B$, entraîner une hypergammaglobulinémie polyclonale et l'apparition d'un second événement nucléaire conduisant à une production monoclonale, responsable d'une cryoglobulinémie et d'une lymphoprolifération maligne $B$ [18].

De part leur nature maligne, le diagnostic précoce est essentiel pour la survie des malades. Dans tous les cas, il repose sur l'examen anatomopathologique d'un prélèvement de tissu tumoral suffisamment important pour permettre une étude morphologique (cytologie et histologie), immunophénotypique, et si possible moléculaire et cytogénétique [3]. Un résultat histopathologique négatif ne permet pas d'exclure définitivement un LNH. Dans une étude portant sur onze cas de lymphome mandibulaire, les auteurs montrent que dans cinq cas, la première biopsie n'avait pas permis de poser le diagnostic de LNH. Cette difficulté diagnostique est due principalement à l'infiltrat inflammatoire qui peut masquer les cellules tumorales et aux lymphoblastes qui sont des cellules très fragiles [12].

Le traitement des LNHE dépend du type histologique et du stade : en général, il comporte une exérèse chirurgicale de la lésion, suivie d'une chimiothérapie et/ou d'une radiothérapie [4]. Pour la chimiothérapie, c'est le plus souvent le protocole " $\mathrm{CHOP}$ », dit de première génération, qui est choisi : il est constitué par de la doxorubicine (50 mg. $\left.\mathrm{m}^{-2}\right)$, du cyclophosphamide (750 mg. $\mathrm{m}^{-2}$ ), de la vincristine $\left(1,4 \mathrm{mg} \cdot \mathrm{m}^{-2}\right)$ et de la prednisone (60 mg. $\mathrm{m}^{-2}$ ). Dans les formes étendues, il associe une phase d'induction délivrant quatre cures espacées de 2 à 3 semaines, suivie d'une phase de consolidation. II est indispensable de délivrer le traitement en respectant les doses prévues et l'intervalle entre les cures, pour obtenir une doseintensité optimale. Parfois il est associé à un facteur de croissance hématopoïétique, comme le granulocyte colony stimulating factor (G-CSF) pour diminuer l'agranulocytose.

Le pronostic des LNH des maxillaires est moins bon que celui des LNH des tissus mous de la cavité buccale. II dépend également du stade de la maladie. Les patients présentant un LNH extraganglionnaire, stade I ou II d'Ann Arbor, ont un taux de survie plus élevé que ceux atteints de LNH ganglionnaires [6].

Le LNH mandibulaire primaire constitue une localisation rare. Le plus souvent, l'atteinte mandibulaire représente une manifestation précoce du développement d'un lymphome ganglionnaire. Dans un cas comme dans l'autre, une douleur dentaire réfractaire à tout traitement constitue le premier signe évocateur. Lorsqu'il existe une incertitude diagnostique, on doit envisager un prélèvement tissulaire pour un examen histopathologique, afin d'exclure un LNH.

Ces deux présentations cliniques illustrent l'intérêt du rôle de l'odontologiste dans le diagnostic précoce car une odontalgie persistante, réfractaire à tout traitement, constitue pendant souvent des semaines, voire des mois, le seul symptôme de la maladie [8]. Les errements diagnostiques résultent souvent d'une démarche incomplète car elle s'intéresse uniquement aux pathologies locales. Ce retard de diagnostic et préjudiciable pour le patient lorsqu'il attend quelques mois. 


\section{RÉFÉRENCES}

1 - Haioun C, Reyes F. Les lymphomes agressifs. Rev Prat $2002 ; 52: 972-7$.

2 - GISSELBRECHT C. Avant propos sur les lymphomes de l'adulte. Rev Prat 2002 ; 52 : 943-4.

3 - LEDERLIN P. Pour la pratique. Lymphomes de l'adulte. Rev Prat $2002 ; 52$ : 991-3.

4 - Mealey BL, Tunder GS, Pemble CW. Primary extranodal malignant lymphoma affecting the periodontium. J Periodontol 2002 ; 73 : 937-41.

5 - Economopoulos T, Asprou N, Stathakis N. Primary extranodal non-Hodgkin's lymphoma in adults. Clinicopathological and survival characteristics. Leuk Lymphoma 1996 ; 21 : 131-6.

6 - Economopoulos T, FountzILAs G, Kostourou A. Primary extranodal non-Hodgkin's lymphoma of the head and neck in adults: A clinicopathological comparison between tonsillar and non-tonsillar lymphomas. Anticancer Res $1998 ; 18$ : 4655-60.

7 - LYNCH DP, CONLON TO, MAXWELL GR, MARTINEZ F. Exophytic gingival mass in a geriatric patient. Oral Surg Oral Med Oral Pathol $1998 ; 85: 5-7$.

8 - SCHIMMING R, LOBNITZER A. Lymphome primaire non hodgkinien de la mandibule, présentation d'un cas. Rev Mens Suisse Odontostomatol 1999 ; 109 : 840-3.

9 - Margiotta V, Franco V, Rizzo A, Porter S, Scully C, DI ALBERTI L. Gastric and gingival localization of mucosaassociated lymphoid tissue (MALT) lymphoma. An immunohistochemical, virological and clinical case report. J Periodontol $1999 ; 70$ : 914-8.

10 - HARRIS NL, JAFFE ES, STEIN H. A revised european-american classification of lymphoid neoplasms: a proposal fro $m$ the international lymphoma study Group. Blood $1994 ; 84: 1361-92$.

11 - Yamada T, Kitagawa Y, Ogasawara T, Yamamoto S, ISHII Y, URASAKI Y. Enlargment of mandibular canal without hypesthesia caused by extranodal non-hodgkin's lymphoma. A case report. Oral Surg Oral Med Oral Pathol $2000 ; 89: 388-92$.

12 - Robbins KT, Fuller LM, Manning J, Goepfert H, VELASQUEZ WS, SUlLIVAN MP. Primary lymphoma of the mandible. Head Neck Surg 1986 ; 8 : 192-9.

13 - Ezzat AA, IBrahim EZ, El Weshi AN, Khafaga YM, Aljurf M, Martin JM, AJarim DS, BazARBash SN, StUART RK, ZUccA E. Localized non Hodgkin's lymphoma of Waldeyer's ring: Clinical features, management, and prognosis of 130 adult patients. Head Neck 2001 : 547-558.

14 - Petrusevska G, Stojanovic A, Karagozov S, Bogoeva B, TolovsKa M, Jovanovic R. Gastrointestinal malignant lymphomas in Macedonia. Acta Med Croatica 2001 ; $55: 153-5$

15 - CARLI PM, MAYNADIE M. Epidémiologie et étiologie des lymphomes non hodgkiniens. Rev Prat 2002 ; 52 : 945-9.

16 - SzPIRGLAS H, LAcoste JP. Manifestations buccales des hémopathies. Enc Med Chir Stomatol 1994 ; 22-050A-10.

17 - Slootweg PJ, WittKampf AR, KLuin PM, De Wilde PC, VAN UNNIK JA. Extranodal non-Hodgkin's lymphoma of the oral tissues. An analysis of 20 cases. J Maxillofac Surg $1985 ; 13: 85-92$.

18 - Besson C, Pialoux G, Mariette X, Lefrere F, Brechot C, HERMINE O. Lymphomes non hodgkiniens et virus de l'hépatite C. Revues et mini-revues - Hématologie $2000 ; 6: 156-63$.

19 - Harzic M, Girard-Pipau F, Halphen M, Ferchal F, Perol Y, Rambaud JC. Etude bactériologique, parasitologique et virologique de la flore digestive dans la maladie des chaînes alpha. Gastroenterol Clin Biol 1985 ; 9 : 472-479.

\begin{tabular}{l}
$\begin{array}{l}\text { médecine } \\
\text { buccale } \\
\text { chirurgie } \\
\text { buccale }\end{array}$ \\
\hline VOL. 12, N N 1 \\
2006 \\
\hline page 29
\end{tabular}

page 29 